\title{
Nanofabrication and characterization of high-line-density $x$-ray transmission gratings
}

\author{
Zhu, Xiaoli; Li, Hailiang; Cao, Leifeng; Liu, Shenye; Shi, Peixiong; Xie, Changqing
}

Published in:

Journal of Micro-nanolithography Mems and Moems

Link to article, DOI:

10.1117/1.JMM.16.3.034503

Publication date:

2017

Document Version

Publisher's PDF, also known as Version of record

Link back to DTU Orbit

Citation (APA):

Zhu, X., Li, H., Cao, L., Liu, S., Shi, P., \& Xie, C. (2017). Nanofabrication and characterization of high-linedensity x-ray transmission gratings. Journal of Micro-nanolithography Mems and Moems, 16(3), 034503. https://doi.org/10.1117/1.JMM.16.3.034503

\section{General rights}

Copyright and moral rights for the publications made accessible in the public portal are retained by the authors and/or other copyright owners and it is a condition of accessing publications that users recognise and abide by the legal requirements associated with these rights.

- Users may download and print one copy of any publication from the public portal for the purpose of private study or research.

- You may not further distribute the material or use it for any profit-making activity or commercial gain

- You may freely distribute the URL identifying the publication in the public portal 


\title{
Micro/Nanolithography, MEMS, and MOEMS
}

\section{Nanofabrication and characterization of high-line-density x-ray transmission gratings}

\author{
Xiaoli Zhu \\ Hailiang Li \\ Leifeng Cao \\ Shenye Liu \\ Peixiong Shi \\ Changqing Xie
}




\title{
Nanofabrication and characterization of high-line-density $\mathrm{x}$-ray transmission gratings
}

\author{
Xiaoli Zhu, ${ }^{a}$ Hailiang Li, ${ }^{a}$ Leifeng Cao, ${ }^{b}$ Shenye Liu, ${ }^{b}$ Peixiong Shi, ${ }^{c}$ and Changqing Xie ${ }^{a, *}$ \\ ${ }^{a}$ Institute of Microelectronics of Chinese Academy of Sciences, Key Laboratory of Microelectronic Devices and Integrated Technology, \\ Beijing, China \\ ${ }^{b}$ China Academy of Engineering, Research Center of Laser Fusion, National Key Laboratory of Laser Fusion, Mianyang, China \\ 'Technical University of Denmark, Danchip-Cen, National Center for Micro- and Nanofabrication, Kgs Lyngby, Denmark
}

\begin{abstract}
We report the nanofabrication and characterization of x-ray transmission gratings with a high aspect ratio and a feature size of down to $65 \mathrm{~nm}$. Two nanofabrication methods, the combination of electron beam and optical lithography and the combination of electron beam, $x$-ray, and optical lithography, are presented in detail. In the former approach, the proximity effect of electron beam lithography based on a thin membrane of low- $z$ material was investigated, and the x-ray transmission gratings with a line density of up to 6666 lines $/ \mathrm{mm}$ were demonstrated. In the latter approach, which is suitable for low volume production, we investigated the $\mathrm{x}$-ray mask pattern correction during the electron beam lithography process and the diffraction effect between the mask and wafer during the x-ray lithography process, and we demonstrated the precise control ability of line width and vertical side-wall profile. A large number of $\mathrm{x}$-ray transmission gratings with a line density of 5000 lines $/ \mathrm{mm}$ and Au absorber thickness of up to $580 \mathrm{~nm}$ were fabricated. The optical characterization results of the fabricated $\mathrm{x}$-ray transmission gratings were given, suggesting that these two reliable approaches also promote the development of x-ray diffractive optical elements. @ 2017 Society of Photo-Optical Instrumentation Engineers (SPIE) [DOI: 10.1117/1.JMM.16.3 .034503]
\end{abstract}

Keywords: Au transmission gratings; electron beam lithography; $x$-ray lithography; $x$-ray spectroscopy.

Paper 17058SS received Apr. 30, 2017; accepted for publication Jul. 25, 2017; published online Aug. 19, 2017.

\section{Introduction}

$\mathrm{Au}$ transmission gratings (TGs) are invaluable optical elements in numerous applications. More specifically, $\mathrm{x}$-ray telescopes such as Chandra and the Solar and Heliospheric Observatory adopted a huge amount of x-ray TGs with a feature size of 100 and $200 \mathrm{~nm}^{1-3}$ Highaspect-ratio $\mathrm{Au}$ TGs were used for hard $\mathrm{x}$-ray differential phase-contrast imaging. ${ }^{4-7}$ In $\mathrm{x}$-ray spectrometers, X-ray TGs with a high line density are key dispersing elements for both spectral and imaging goals..$^{8-11}$ The heavy demands of x-ray TGs in scientific instruments with a compact size, light weight, and high degree of design flexibility have motivated considerable research on nanofabrication techniques. Various performance goals of x-ray TGs pose an extreme challenge to nanofabrication technologies.

Currently, the methods for manufacturing high-linedensity gratings include interference lithography plus deep reactive ion etching, ${ }^{12,13}$ interference lithography followed by anisotropic Si wet etching, ${ }^{14,15}$ nanoimprint and multistep etching, ${ }^{16}$ ion beam lithography, ${ }^{17,18}$ etc. In addition, electron beam lithography (EBL) and its combination with other nanofabrication technologies are of great interest for fabricating x-ray diffractive optical elements including X-ray TGs. ${ }^{9-21}$ EBL tools have been developed with a fast $100-\mathrm{MHz}$ line frequency and a beam diameter of several nanometers. ${ }^{22} \mathrm{~A}$ remarkable progress has also been made in which nanostructures with a high aspect ratio of up to $20 \mathrm{~nm}$ and patterns with 4.5-nm half-pitch have been

*Address all correspondence to: Changqing Xie, E-mail: xiechangqing@ime.ac .cn achieved by a single EBL step. ${ }^{23-25}$ For many specific applications, EBL continues its path to obtain features with smaller dimensions and higher aspect ratios and can be combined with other lithographic technologies to realize the desired elements. ${ }^{26}$ However, every above-mentioned lithographic method has strengths and weaknesses and still suffers from many challenges such as sophisticated deepreactive-ion etching process, mask fabrication, and low writing speed. ${ }^{12,16,19}$

Here, we report on our recent progress on nanofabrication and characterization of high-line-density x-ray TGs. On one hand, to fabricate 6666 lines/mm x-ray TGs, EBL was used to directly write patterns on the membrane of low- $z$ material and combined with ultraviolet lithography (UVL) to define supporting frames. To meet the heavy demands of $\mathrm{x}$-ray TGs with 5000 lines/mm density, $100 \mathrm{~nm}$ bar width, and $580 \mathrm{~nm}$ height, EBL, x-ray lithography (XRL) and UVL were combined to fabricate TGs in low volume production. Two combined approaches are capable of directly patterning nanoscale resist structures with a vertical cross section, avoiding the sophisticated etching process. Both of them are robust fabrication procedures under tight process control with a large process latitude. The former is able to fabricate finer gratings, and the later has higher yields. For the individual combination, to precisely control the line/space ratio and achieve a vertical cross section, some improvements have to be made such as writing patterns on membrane substrates in EBL, pattern correction on the mask, and mask/ wafer gap control in XRL. The subsequent characterization 
of fabricated x-ray TGs has shown their satisfying optical performances.

\section{Design of Self-Supporting X-Ray TGs}

Figure 1 shows the schematic geometry of x-ray TGs, consisting of free-standing gold grating bars with a width of down to several tens of nanometers supported by coarse mesh or polyimide membranes. The diagram does not show proper proportions. The diameter of the supporting silicon frame is $20 \mathrm{~mm}$, and the grating area is from several to a few tens of square millimeters, which can be patterned by direct-writing electron beam lithographic tools. In the soft $\mathrm{x}$-ray region from 0.1 to $2 \mathrm{keV}, \mathrm{x}$-ray is intensively absorbed in any known material. ${ }^{27,28}$ Therefore, $\mathrm{x}$-ray must freely pass through gaps between gratings bars without any absorption. In other applications where photon energy is above $2 \mathrm{keV}$, grating bars can be fabricated on x-ray transparent membranes of low- $z$ materials. ${ }^{29}$ The refraction index of $x$-ray in any material can be written as $n=1-\gamma-i \beta$, where $\gamma$ and $\beta$ are very small for all materials, resulting in much difficulty in deflecting $x$-rays and designing $\mathrm{x}$-ray diffractive optical elements. ${ }^{30}$ When x-rays pass through fine structures of high- $z$ materials, both the intensity and phase are modulated simutaniously ${ }^{31,32}$ offering a multifunctional and flexible way to diffract or focus $\mathrm{X}$-rays.

Spectral performance of gratings is mainly determined by two factors: the period and the absorber thickness. ${ }^{37}$ At normal incidence, the first-order diffraction angle of the grating can be expressed as

$\theta=\sin ^{-1}(\lambda / p)$,

where $\theta$ is the diffraction angle, $\lambda$ is the wavelength of incident light, and $p$ denotes the grating period. ${ }^{33}$ To obtain a large diffraction angle (i.e., high spectral resolution), one needs to decrease the period of TGs to $150 \mathrm{~nm}$ for their applications in high spectral-resolution spectrometers.

Another crucial parameter of $x$-ray TGs is the efficiency, especially for their applications in $\mathrm{x}$-ray spectrometers. The diffraction efficiency of wire gratings with a rectangular cross section can be calculated as ${ }^{34,35}$
chaction

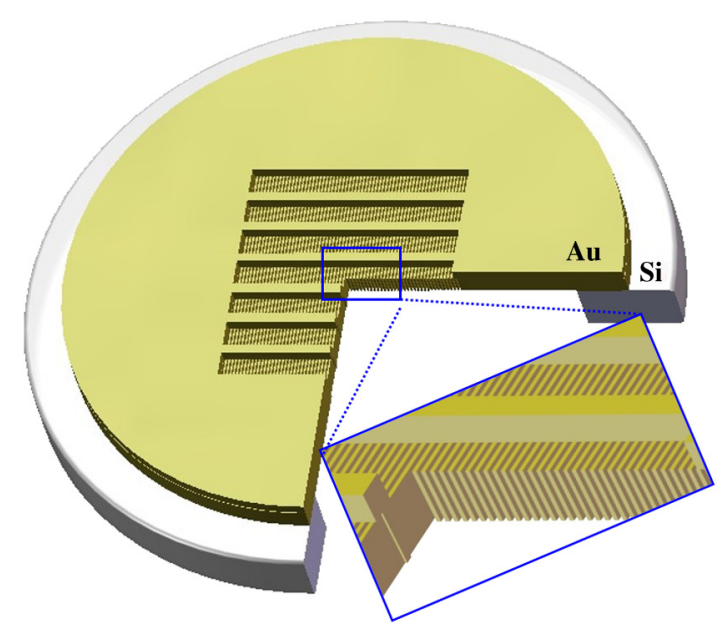

Fig. 1 Schematic geometry of $x$-ray TGs with free-standing Au grating bars supported by coarse gold frames. $\eta_{m}(\lambda)=\left[\frac{\sin \left(\frac{m \pi a}{p}\right)}{m \pi}\right]^{2}\left[1+e^{-\frac{4 \pi}{\lambda} \beta z}-2 e^{-\frac{2 \pi}{\lambda} \beta z} \cos \left(\frac{2 \pi}{\lambda} \delta z\right)\right]$,

where $m \neq 0, a$ is the gap between grating bars, $z$ is the absorber thickness, $\lambda$ is the wavelength of incident $\mathrm{x}$-ray light, $\beta$ is the imaginary part of the refractive index, and $\delta$ is the real part of the refractive index. On one hand, it can be deduced that when the ratio of gap to period (i.e., duty cycle) is 0.5 , the first-order efficiency reaches a maximum, and all even orders disappear. If the duty cycle is up to 0.75 or down to 0.25 , the efficiency decreases to $50 \%$ of the maximum. On the other hand, the efficiency of x-ray TGs is also determined by the thickness of gratings. According to Eq. (2), the efficiency approaches its maximum when $z$ is $\lambda /(2 \delta)$ and the phase shift is correspondingly $\pi$. To achieve enough diffraction efficiency, 300 to $600 \mathrm{~nm}$ of bar height is required in the x-ray region. In addition, the diffraction efficiency decreases when the cross section deviates from the rectangle. ${ }^{36}$

To achieve high spectral resolution, enough efficiency, and desired phase shift, nanoscale grating bars with nanoscale width, desired height of absorber, and precisely controlled line/space ratio must be constructed to be freestanding or on membranes of low- $z$ materials.

\section{High-Line-Density X-Ray Transmission Gratings Fabricated by Electron Beam Direct Writing}

\subsection{State-of-Art of X-Ray TGs Fabrication}

Until now, a variety of lithographic techniques have been developed to realize design targets of $\mathrm{x}$-ray TGs. Both advantages and tradeoffs exist for each lithographic technique. Researchers at Massachusetts Institute of Technology and Max-Planck-Institut fur Physik und Astrophysik (Germany) have accomplished the pioneering work and developed a huge amount of x-ray TGs with bar widths of 100 and $200 \mathrm{~nm}$ using interference lithography. ${ }^{37-40}$ This process can also be followed by wet anisotropic etching to achieve X-ray critical point TGs with extremely high aspect ratios. ${ }^{41,42}$ Extreme ultraviolet (EUV) interference lithography and its variants can scale the EUV gratings pitch down to $50 \mathrm{~nm}$ and fabricate periodic structures smaller than $10 \mathrm{~nm}$, but they suffer from the limitation of periodic patterns and uniformity in large areas. ${ }^{43-47}$ Nanoimprint provides a low-cost approach to fabricating gratings with high spatial resolution. However, for many applications, there are still many challenges such as defects and template patterning. ${ }^{48,49}$ In recent years, anisotropic $\mathrm{Si}$ wet etching in hydrofluoric acid and hydrogen peroxide solutions enables to achieve silicon nanostructures with an extremely high aspect ratio of up to 120 , but how to transfer patterns from silicon to metal is challenging. ${ }^{50}$

In addition, EBL has shown its potential in fabricating of high-line-density x-ray TGs due to its high resolution and large penetration depth in the resist. An 100-keV electron beam writer can directly pattern gold gratings with $1 \mu \mathrm{m}$ height and $45 \mathrm{~nm}$ width. ${ }^{20}$ 50-nm-wide and 300-nm-thick platinum grating structures were fabricated from a trilayer resist exposed by EBL and followed by reactive ion etching plus electroplating. ${ }^{21}$ The beam size can be focused within 
several nanometers to fabricate EUV TGs with a lines density of 10,000 lines/mm and an absorber height of $80 \mathrm{~nm}^{27}$ Although EBL is capable of patterning gratings with line density of up to 10,000 lines/mm in the EUV region, the thickness of the absorber is several tens of nanometers. Moreover, how to combine EBL with other lithographic techniques to manufacture $\mathrm{x}$-ray TGs in high throughput has been challenging for several decades. ${ }^{38}$ Furthermore, EBL can generate arbitrary patterns for more flexible designs including variable gratings and binary optical elements. ${ }^{51}$ To date, the nanofabrication of high-line-density x-ray TGs operating in the $\mathrm{x}$-ray region is still technically challenging.

\subsection{Combination of EBL and Optical Lithography}

The desired $6666 \mathrm{l} / \mathrm{mm} \mathrm{x}$-ray TGs consist of grating bars with a $75 \mathrm{~nm}$ half-pitch and a $350 \mathrm{~nm}$ thickness on a $2.0-\mu \mathrm{m}$-thick polyimide membrane that is $\mathrm{x}$-ray transparent in a $2.0-$ to $4.0-\mathrm{keV}$ region. In the fabrication process, the combination of EBL and UVL was adopted to pattern the grating structures. EBL can be performed to directly pattern grating structures, taking advantages of extremely high resolution, acceptable writing speed, and large penetration depth of electrons. To our knowledge, few works have been able to realize $\mathrm{x}$-ray TGs with line density of up to 6666 lines $/ \mathrm{mm}$ that can operate at a photon energy of up to $4 \mathrm{keV}$.

The process of the combination of EBL and UVL, which is shown in Fig. 2, consists of two main steps in which EBL was used to pattern nanoscale gratings and UVL was performed to generate coarse supporting frames. First, the process started by depositing of 15-nm $\mathrm{CrAu}$ on a polyimide membrane using electron evaporation; second, an extremely fine electron beam with a $100 \mathrm{kV}$ accelerating voltage and a 200 pA current (JBX6300FS, JEOL) was used to directly write patterns in polymethylmethacrylate (PMMA, 5\% 2200k in anisole, resist 1 in Fig. 2) resist, followed by gold electroplating in the gaps of the resist structures. Third, to define supporting frames on the grating structures, a PMMAbased resist of SX AR-N 4800/16 (resist 2 in Fig. 2) was exposed by an aligner, developed in the mixture of methylisobutylketone (MIBK) and isopropyl alcohol (IPA), and rinsed in IPA. Fourth, electroplating was performed to form coarse supporting gold fames. Finally, x-ray TGs were

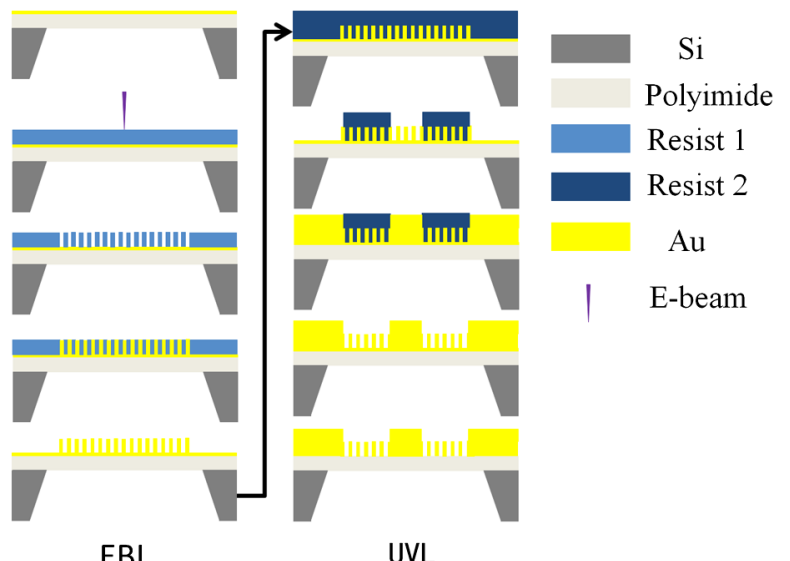

Fig. 2 The combined process of EBL and UVL for fabricating x-ray TGs. finished after removing the $\mathrm{CrAu}$ seed layer by argon plasma etching.

In the above processes, PMMA coated on the polyimide membrane was exposed by an electron beam writer with $100 \mathrm{kV}$ accelerating voltage to decrease the proximity effect due to two factors. One is that the membrane substrate can effectively decrease the extradose caused by backscattered electrons. Another is that the accelerating voltage of $100 \mathrm{kV}$ makes it possible to prevent the obvious beam size broadening due to little forward scattering. ${ }^{52}$ To demonstrate the proximity effect in PMMA resist and the membrane, the distribution of absorbed energy in 400-nm PMMA on a polyimide membrane coated with 5-nm $\mathrm{Cr}$ and 10-nm $\mathrm{Au}$ was calculated by the Monte Carlo simulation method (Tracer, GenIsys). In the simulation, electron beam energy of $100 \mathrm{keV}$ and the electron number of $10^{6}$ were used. Figure 3 shows the radial distribution of energy density absorbed in 400-nm PMMA, 15-nm CrAu seed layer, and $2-\mu \mathrm{m}$ polyimide membrane. The embedded zoom-in image in the bottom-left shows the energy distribution near the incident point. In the radial direction, when the energy density decreases by two orders, the widths of energy distribution from the zero point on the top and bottom of the resist are 2.37 and $2.48 \mathrm{~nm}$, respectively. The simulation result demonstrates the ability to pattern high-line-density gratings with a vertical cross section and high aspect ratio, both of which are crucial in the nanofabrication of $\mathrm{x}$-ray TGs.

By exposing 400-nm PMMA resist on a $2-\mu$ m polyimide membrane, extremely high contrast of resist can be achieved in exposure. During the electron beam exposure, the calibration of electron beam was automatically carried out every 5 min to avoid the writing field distortion caused by the surface height fluctuation. After development and electroplating, the fabricated grating structures are shown in Fig. 4(a). In addition, the polyimide membrane was used to support grating structures due to its robustness and excellent radiation resistance. However, during UVL, the polyimide membrane becomes wrinkled, and patterns on the membranes get distorted when polyimide membrane is immersed into alkaline-based developer. Thus, SX AR-N 4800/16 negative resist was used to define coarse supporting frames since it can be dissolved in an organic developer mixed by MIBK and IPA, which do not lead to wrinkled polyimide

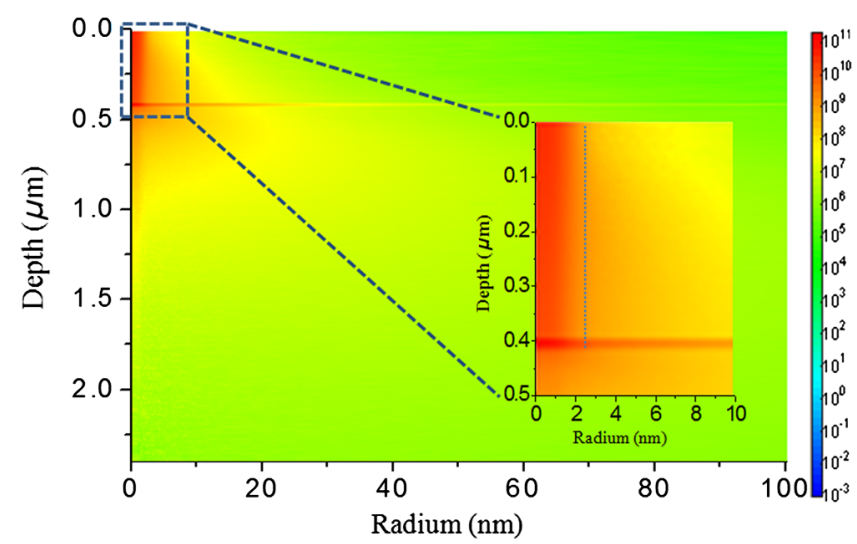

Fig. 3 Simulated radial energy density absorbed in the stack of 400-nm PMMA/10-nm Au/5-nm Cr/2- $\mu$ m polyimide, the energy of incident electrons is $100 \mathrm{keV}$. 


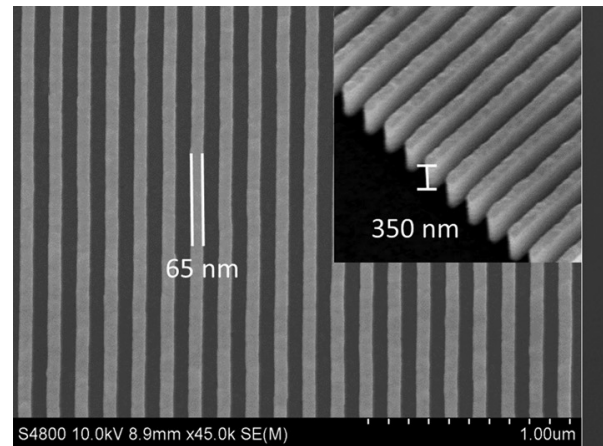

(a)

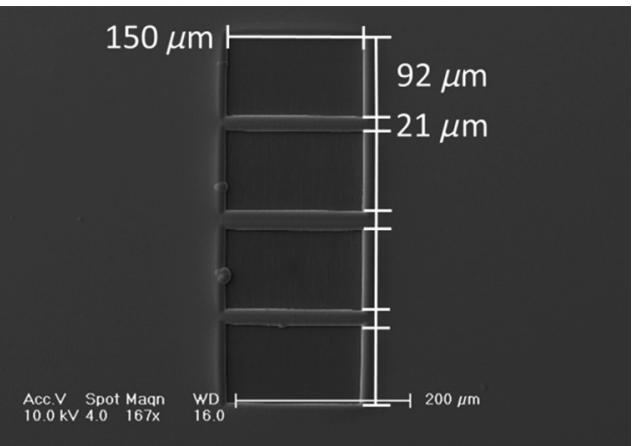

(b)

Fig. 4 Achieved high-line-density x-ray TGs consisting of (a) grating bars fabricated by EBL and electroplating and (b) coarse frame defined by UVL with SX AR-N 4800/16 negative resist.

membrane. The UVL was performed to pattern the coarse meshes on the grating bars, and the overlay accuracy of EBL and UVL was kept within $2 \mu \mathrm{m}$. After exposure, the resist structures were transferred to the Au layer by electroplating. Finally, the achieved device is shown in Fig. 4(b). Grating bars are perpendicular to the supporting bars. Every opening area of grating is $150 \mu \mathrm{m} \times 92 \mu \mathrm{m}$ and supported by $21-\mu \mathrm{m}$ wide bars. The grating area patterned by EBL is $450 \mu \mathrm{m} \times 92 \mu \mathrm{m}$, and the writing time was $15 \mathrm{~min}$. The area could be several times larger if the trade-off between electron current and beam diameter has been made to optimize the lateral resolution and the writing speed. In addition, in the final step, the $\mathrm{CrAu}$ seed layer was removed by a maskless process of induced plasma coupling etching. Using a high platen power, the 15-nm-thick $\mathrm{CrAu}$ seed layer was etched and the width of the gold bars was hardly changed.

\subsection{Characterization of 6666 Lines/mm X-Ray TGs}

The diffraction pattern of fabricated 6666 lines/mm x-ray TGs was obtained at a 4B7A beamline on a Beijing synchrotron radiation facility, which covers the photon energy range from 2 to $6 \mathrm{keV}$. The incident $\mathrm{x}$-rays passed through the shutter and the $\mathrm{x}$-ray TGs and reached the detector. Behind the sample, an x-ray charge-coupled device with $2048 \times 2048$ pixels and $13.5 \mu \mathrm{m}$ pixel size was used to receive the diffraction pattern. Figure 5(a) shows the diffraction pattern that is symmetrically distributed on both sides of the zeroth-order when the photon energy of incident x-ray was $2.9 \mathrm{keV}$. The second-order cannot be neglected, while any third-order is not visible. This is due to the deviation of the grating bar width from the original design. The measured grating bar width is $65 \mathrm{~nm}$ rather than the desired $75 \mathrm{~nm}$ in Fig. 4(a). Figure 5(b) shows the measured ratio of first-order to zeroth (i.e., relative diffraction efficiency) at an energy range of 2.1 to $4.0 \mathrm{keV}$. The relative firstorder efficiency is from $8.89 \%$ to $9.41 \%$, which is acceptable for $150-\mathrm{nm}$ period $\mathrm{x}$-ray TGs operating at $4.0 \mathrm{keV}$. Because of the $350 \mathrm{~nm}$ thickness of the absorber, the efficiency gradually decreases at photon energy above $3.5 \mathrm{keV}$.

\section{X-Ray TGs Nanofabrication in High Throughput}

\subsection{Combination of $E B L, X R L$, and $U V L$}

Although EBL can generate patterns with extremely high spatial resolution and a high aspect ratio, it suffers from its serial writing strategy. The fabrication process of $x$-ray TGs is very time-consuming, and membrane-based $\mathrm{x}$-ray TGs are definitely fragile in their characterization and use. To meet the heavy demands of X-ray TGs in their applications, the combination of EBL, XRL, and UVL was developed to manufacture nanostructures in high throughput. ${ }^{54-56}$ EBL has the ability to pattern structures with less than 5-nm

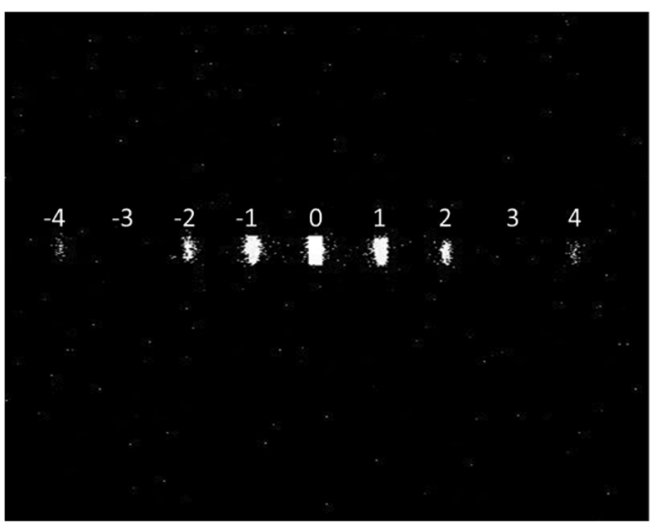

(a)

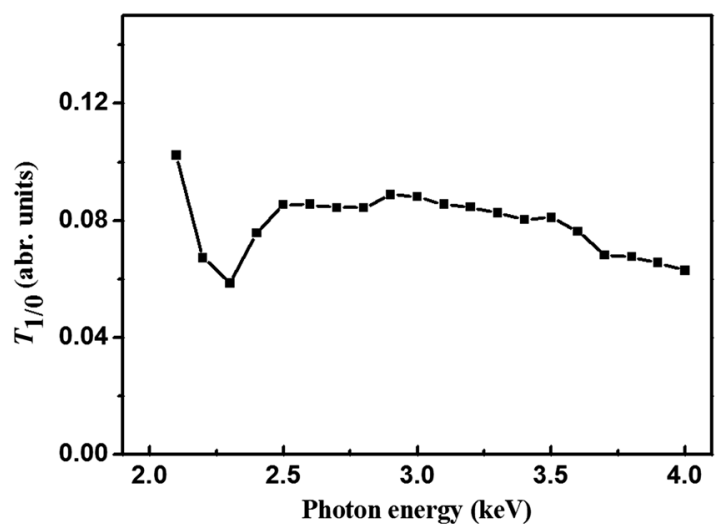

(b)

Fig. 5 Characterization results of 6666 lines/mm x-ray TGs, including (a) diffracted pattern at $2.9 \mathrm{keV}$ and (b) the measured relative first-order efficiency in $2.1-$ to $4.0-\mathrm{keV}$ energy range. 
critical dimension and generate arbitrary complex patterns. XRL enables the efficient replication of nanostructures with a sub-20-nm spatial resolution or a high aspect ratio due to the short wavelength, large depth of focus, and low $\mathrm{X}$-ray absorption in resist. ${ }^{57}$ Although the fabrication of XRL mask by EBL is costly and time consuming, once one or two masks have been finished, a large number of specimens with a high aspect ratio in high fidelity can be promptly achieved without sophisticated induced coupling plasma etching.

To fabricate X-ray TGs in high throughput, the combination of EBL, XRL, and UVL was developed to fabricate 5000 lines/mm x-ray TGs, as shown in Fig. 6. First, in the EBL process, a scanning electron beam with a diameter of several nanometers was focused to pattern resist structures with low aspect ratios on silicon nitride or silicon carbide membranes covered by $\mathrm{CrAu}$ seed layers. After exposure, $\mathrm{Au}$ gratings were formed through spaces between resist structures. Second, with the mask fabricated in the EBL process, XRL and electroplating were formed to replicate nanostructures with high aspect ratios on resist/AuCr/polyimide/Si stacks. Third, in the UVL step, an aligner and $\mathrm{Au}$ electroplating were performed to pattern supporting frames. Finally, the backside silicon of substrate was removed by fluoric acid-based solution; the seed layer and polyimide were removed by argon and oxygen plasma, respectively.

\subsection{Pattern Correction of Master Mask}

Before the replication of $x$-ray TGs by XRL, the EBL step started by vapor-coating a 5-nm $\mathrm{Cr}$ layer and a 10-nm $\mathrm{Au}$ layer as a plating base on $2-\mu$ m-thick $\mathrm{Si}_{3} \mathrm{~N}_{4}$ membranes. The resist of 400-nm-thick PMMA (5\% 2200k in anisole) was spin-coated and baked at $160^{\circ} \mathrm{C}$ for $30 \mathrm{~min}$ in an oven. Then, the resist was exposed using a $100-\mathrm{kV}$ electron beam writer. The beam current was $0.2-\mathrm{nA}$, and the dose was $1800 \mu \mathrm{C} / \mathrm{cm}^{2}$ (JBX6300FS, JEOL). The length and width of grating area were 10 and $0.1 \mathrm{~mm}$, respectively. Resist patterns with an aspect ratio of 4 were obtained after development in MIBK: IPA (1:3) for $40 \mathrm{~s}$ and rinsed in IPA for $30 \mathrm{~s}$. The 350-nm-thick gold structures were formed by a pulse reverse electroplating process in a gold sulfite solution

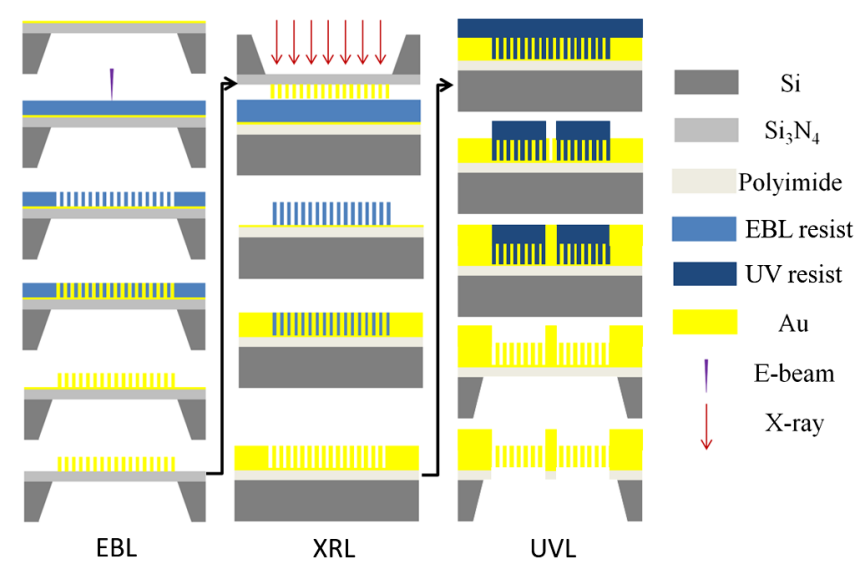

Fig. 6 Process flow to fabricate $x$-ray TGs by the combined process of EBL, XRL, and UVL. The first part contains e-beam evaporation seed layer on $\mathrm{Si}_{3} \mathrm{~N}_{4}$ membrane, electron beam exposure, electroplating, and removal of the resist and seed layer. The second part includes $\mathrm{XRL}$, electroplating, resist removal, and backside silicon wet etching. The third part consists of UVL, electroplating, and etching of silicon, seed layer, and polyimide.
(Elevate gold RTU 7790). After that, PMMA was removed in an acetone solution, and the seed layer was etched by argon plasma. To get a stable process in EBL, electrons were focused to expose the resist on the $\mathrm{Si}_{3} \mathrm{~N}_{4}$ membrane, which can eliminate the backscattering effect similar to that on polyimide membrane demonstrated in Sec. 3.2. Compared with backscattered electrons on bulk silicon substrates, more electrons pass through the membrane substrate and less are scattered by the atoms in the substrate.

To achieve nanoscale Au grating bars with equal widths and gaps using XRL, pattern correction must be considered when starting the fabrication of x-ray mask the EBL step. Based on previous empirical experience and theoretical analysis (presented in Sec. 4.3), to pattern grating bars with a width of 75 to $100 \mathrm{~nm}, 5$ to $10 \mathrm{~nm}$ bias of the grating bar on the mask for XRL is needed. While for 150- to 250$\mathrm{nm}$ wide grating bars, $25 \mathrm{~nm}$ bias of bar width on the mask for XRL is suitable. For example, grating structures of 110-nm wide lines and 90-nm wide spaces on the mask are required for replicating 100-nm wide line/space gratings by XRL. The difference of bias for bars with different widths is mainly determined by the diffraction effect when x-rays pass through the mask/wafer. In EBL, to achieve an optimal line/ space ratio, the resist was exposed with different line-spaceratio patterns on the layouts at different doses. By exposing layout patterns with $90-\mathrm{nm}$ line and $110-\mathrm{nm}$ spacing at $1800 \mu \mathrm{C} / \mathrm{cm}^{2}$ dose, the desired grating patterns were achieved, as shown in Fig. 7, with the line width of $110 \mathrm{~nm}$ on the mask. The bias of grating bars was precisely kept as $10 \mathrm{~nm}$. Generally, the error of bar width must be within $\pm 5 \mathrm{~nm}$, which will benefit the replication of gratings with equal widths of bars and gaps in the following XRL step.

\subsection{Simulation of Pattern Formation in $X R L$}

Compared with other lithographic techniques, XRL has advantages of high resolution, large depth of focus, insensitivity to different substrates, etc. However, in printing 100$\mathrm{nm}$-wide gratings, the variation of patterns on the mask and the substrate cannot be ignored since the diffraction effect between the mask and wafer becomes more evident with smaller critical dimensions or larger mask/wafer gaps. ${ }^{58,59}$ Hence, pattern correction of the $\mathrm{x}$-ray mask must be considered. ${ }^{53}$ To predict the pattern variation at different mask/wafer gaps, the propagation of x-ray between mask and wafer was simulated by a one-dimensional beam-propagating-method program with fast Fourier transformation (1-D BPM-FFT). The simulation consists of $x$-ray propagation, $\mathrm{X}$-ray Fresnel diffraction, and resist blur. In the simulation, width and period of bars on the mask are 110 and $200 \mathrm{~nm}$, respectively. The blur of resist is $10 \mathrm{~nm}$, and the working wavelength is $1 \mathrm{~nm}$. Aerial image profiles with different gaps on the resist surface are shown in Fig. 8. The full width at full maximum (FWHM) of lateral intensity distributions with mask/wafer gaps from 1 to $5 \mu \mathrm{m}$ are 95,85 , 79 , and $55 \mathrm{~nm}$, respectively. The FWHM is changed from $95 \mathrm{~nm}$ at $1 \mu \mathrm{m}$ gap to $79 \mathrm{~nm}$ at $3 \mu \mathrm{m}$ gap, demonstrating that XRL has the potential to pattern high aspect ratio structures with $100 \mathrm{~nm}$ feature size and $2 \mu \mathrm{m}$ height. In the exposure, due to the transportation of secondary electrons and photoelectrons, the width of exposed bars will be broadened by 10 to $20 \mathrm{~nm}$, which can partially compensate for the pattern shrinkage caused by the diffraction of $\mathrm{x}$-rays between 


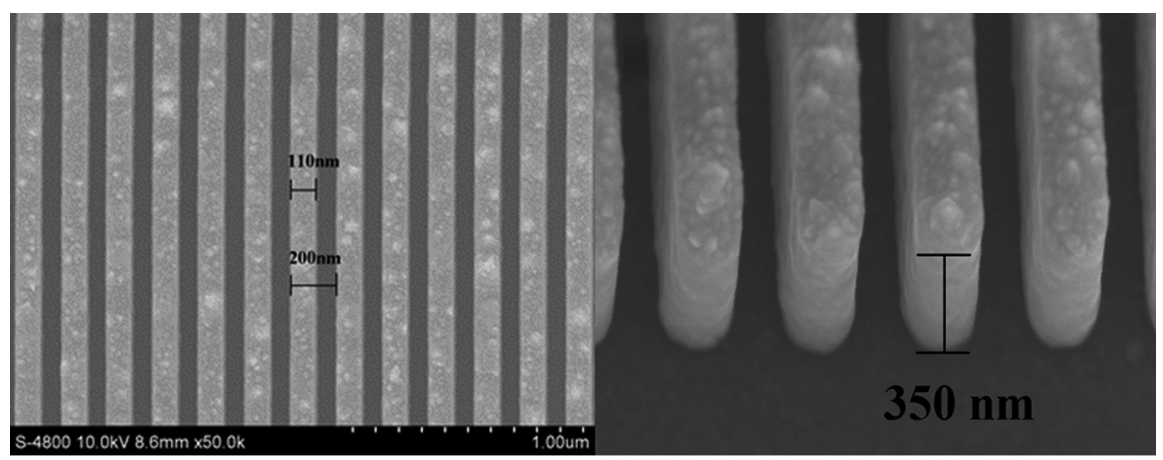

(a)

(b)

Fig. 7 Patterns with $10 \mathrm{~nm}$ bias on the master mask taken from (a) top view and (b) cross section tilted at 30 deg.

the mask and wafer. The above analysis indicates that, to replicate grating bars with 100-nm line/space, $110 \mathrm{~nm}$ width of bars on the mask is required and the gap of mask/wafer must be less than $3 \mu \mathrm{m}$. In the experimental setup, a magnetic force was applied on the mask/wafer holder to achieve a gap of less than $3 \mu \mathrm{m}$, which can be detected by counting Moire rings on the backside $\mathrm{Si}_{3} \mathrm{~N}_{4}$ membranes.

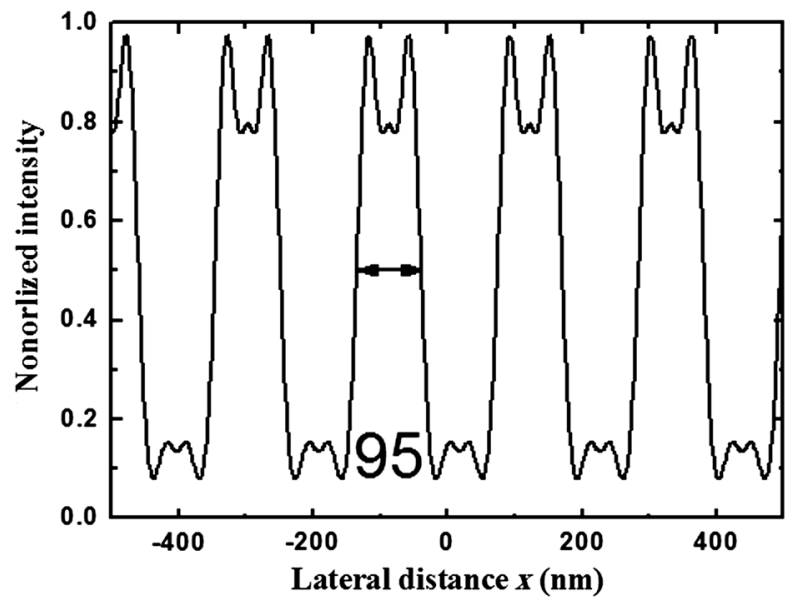

(a)

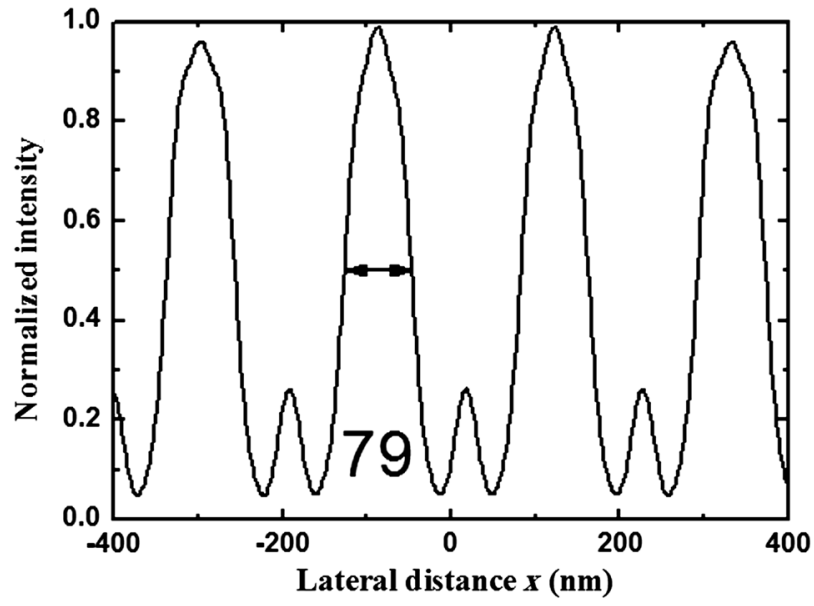

(c)

\subsection{Replication of Gratings with High Aspect Ratio}

The replication processes are very similar to those for fabricating $\mathrm{x}$-ray masks in the EBL step, as shown in Fig. 6. A 600 -nm-thick PMMA resist coated on $2-\mu$ m-thick polyimide film with plating base was exposed at a dose range of 6 to $8 \mathrm{~J} / \mathrm{cm}^{2}$, developed in MIBK: IPA $(1: 3)$ for $40 \mathrm{~s}$ and rinsed in IPA for $40 \mathrm{~s}$; resist patterns were transferred to a

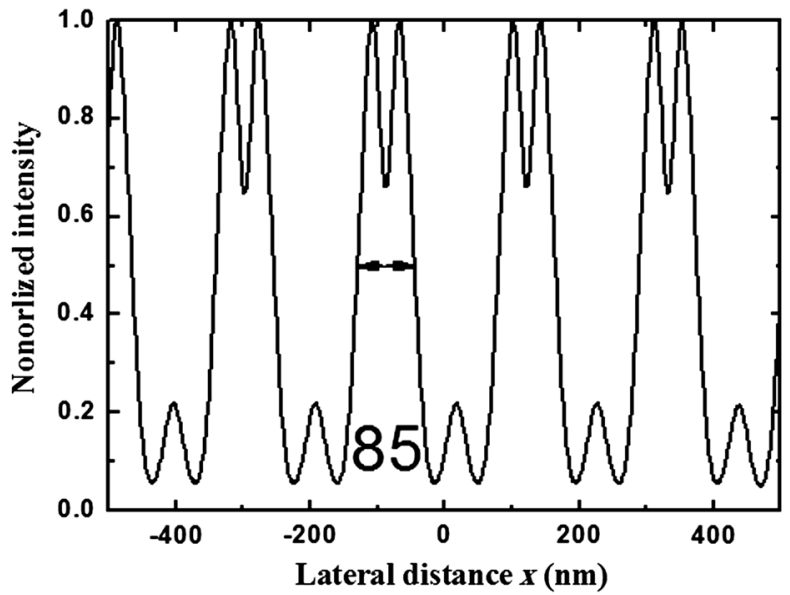

(b)

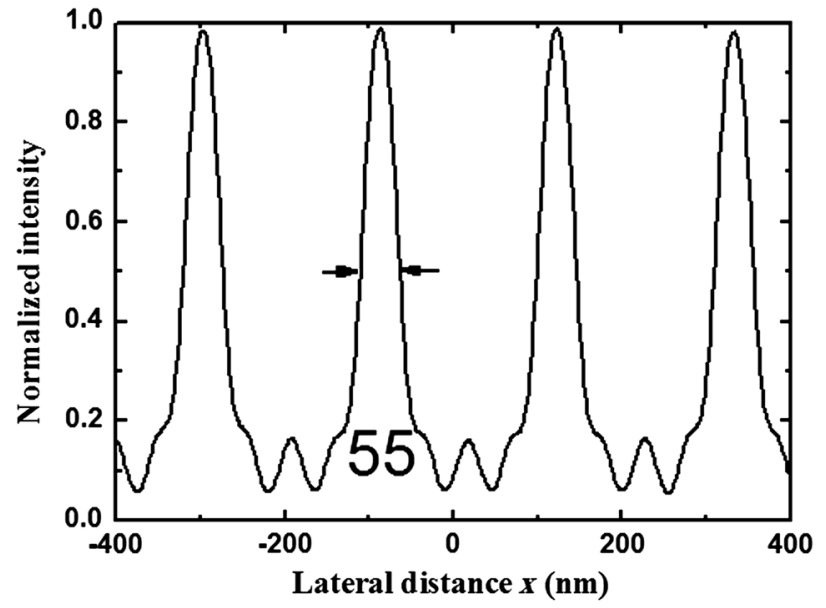

(d)

Fig. 8 Simulated aerial image profiles with different mask/wafer gaps of (a) 1, (b) 2, (c) 3, and (d) $5 \mu \mathrm{m}$, respectively. 


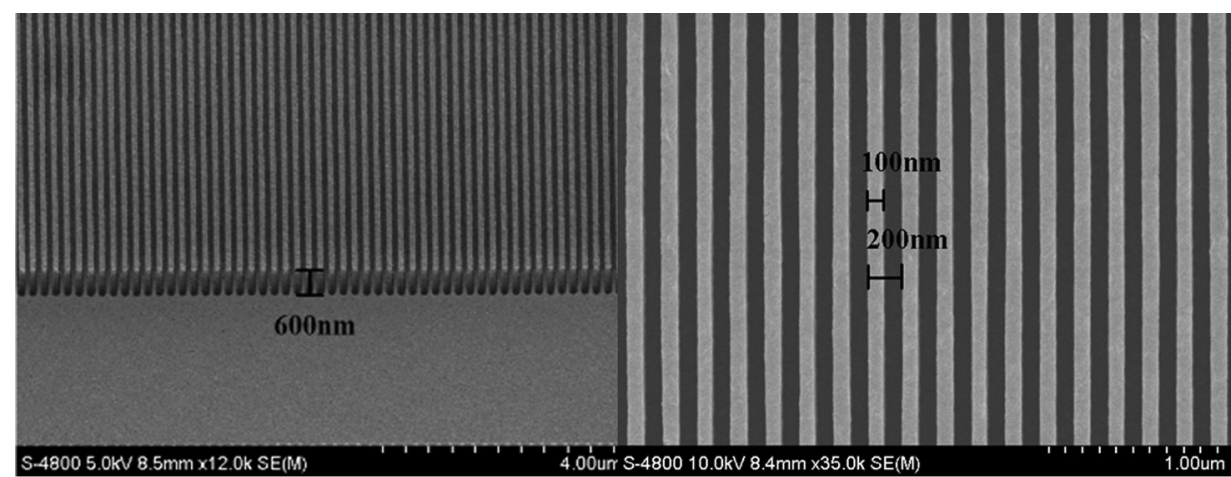

(a)

(b)

Fig. 9 Replicated grating patterns including (a) resist patterns with rectangular cross section and (b) smooth gold gratings with equal widths of bar and gap.

gold layer using electroplating. After that, UVL was carried out to define coarse supporting frames on the grating structures with the overlay accuracy of $2 \mu \mathrm{m}$ and was followed by Au electroplating. Finally, bulk silicon on the backside was etched by the solution of hydrofluoric, nitric, and acetic acids. The replicated gratings with equal bar and gap widths, smooth edges, and rectangular cross section are shown in Fig. 9. The gold thickness in Fig. 9(b) is $580 \mathrm{~nm}$.

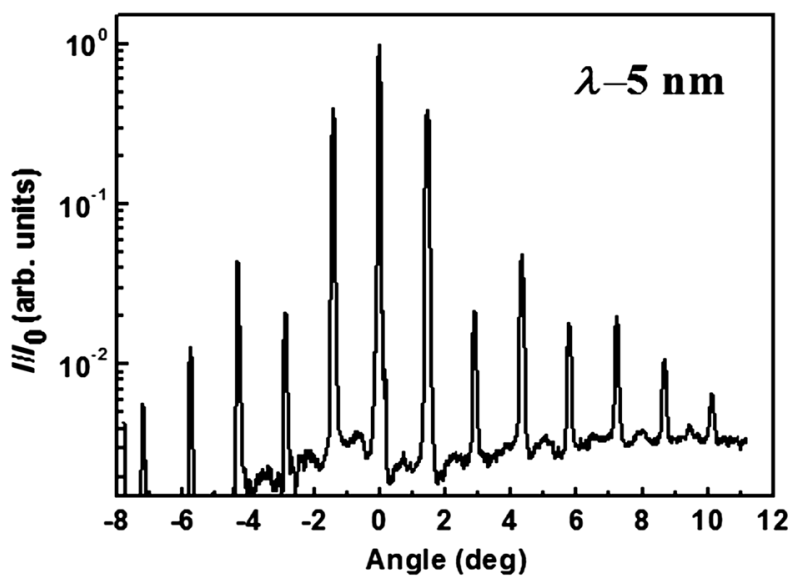

(a)

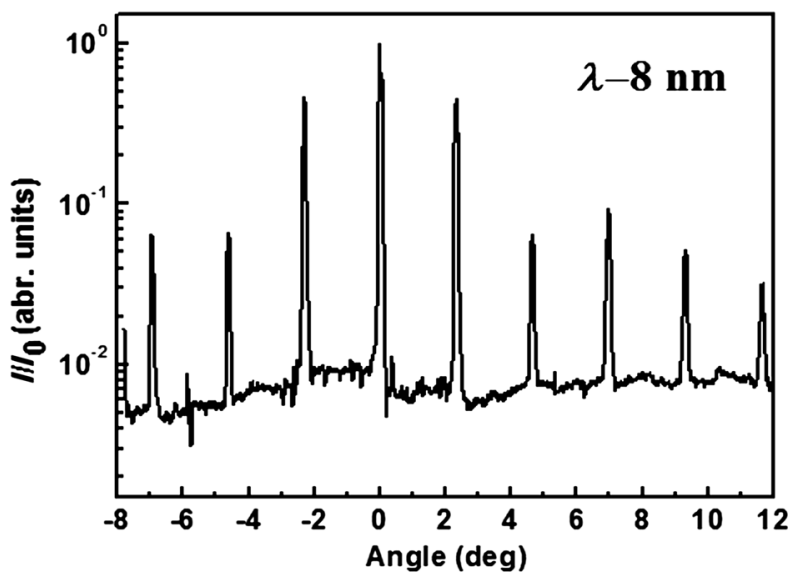

(c)
To form x-ray grating bar gold structures, electroplating rather than the lift-off process is more frequently used because electroplating is able to transfer the resist pattern to thicker metal layer than lift-off. In the above process, no adhesion layer was used to stabilize the lithographic process, since an adhesion layer can frequently lead to failure in the following process. In this combined process, the pattern generation process is straightforward and robust. The

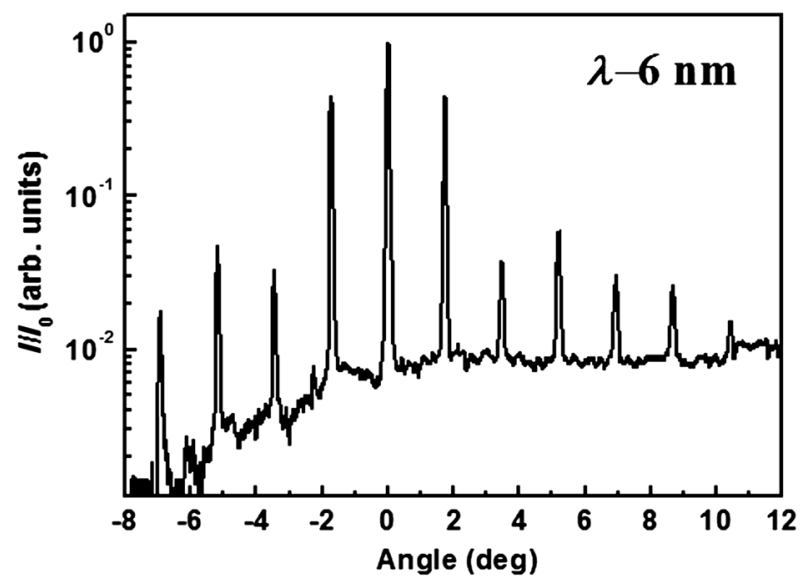

(b)

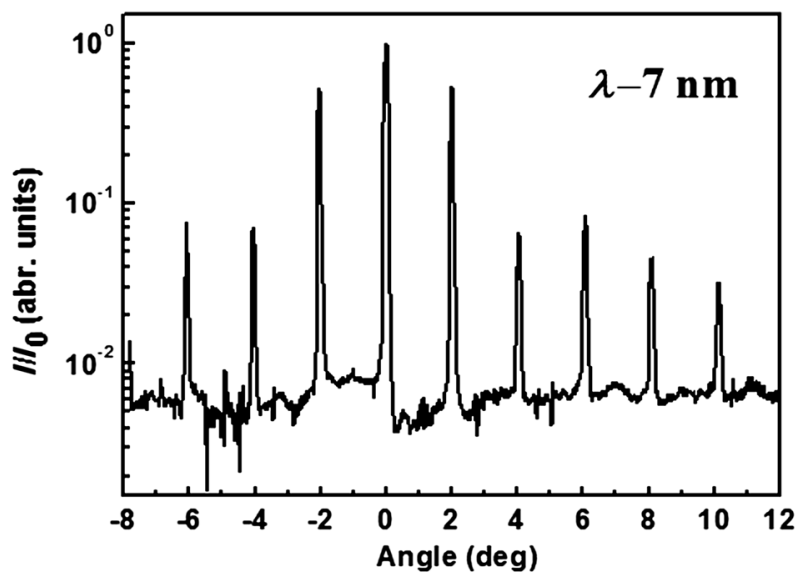

(d)

Fig. 10 Measured angular distribution of spectra at different wavelengths of (a) 5, (b) 6, (c) 7, and (d) $8 \mathrm{~nm}$, respectively. 
combined process is able to produce a large number of x-ray TGs within a limited period for heavy demands. Moreover, due to extremely large penetration depth of x-rays in exposure, it can generate nanopatterns with a high aspect ratio and rectangular cross section, which are desired in various $\mathrm{x}$-ray diffractive optical elements.

\subsection{Characterization of Replicated 5000 Lines $/ \mathrm{mm}$ $X$-Ray TGs}

The characterization of replicated $\mathrm{x}$-ray TGs was performed at U26 beamline, Hefei Synchrotron Radiation Light Source. The geometric position of gratings was controlled by a sample holder with $0.005 \mathrm{deg}$ accuracy between the Si photodiode detector and filter plus slit. X-rays from the storage ring were selected by the monochromator gratings (600 and 1800 lines $/ \mathrm{mm}$ ), and the contribution of higher diffraction orders was limited by setting different filters. The $100-\mu \mathrm{m}$ slit was used to collimate the light on the x-ray TGs, and all measurements were performed with monochromatic radiation normal to the gratings.

The obtained spectra at different wavelengths are shown in Figs. 10(a)-10(d). The ratio of different order efficiencies can be formulated as $T_{m / n}$. The measured $T_{1 / 0}, T_{2 / 1}$, and $T_{3 / 1}$ are $51.3 \%, 6.3 \%$, and $13.8 \%$, respectively. $T_{2 / 1}$ is much smaller than $T_{3 / 1}$, demonstrating that the line/space ratio was precisely controlled as $1: 1$. From the spectra at different wavelengths, the value of $a / d$ was developed as 0.44 , which is definitely close to the ideal value of 0.5 compared with the measured data of X-ray TGs fabricated by interfering lithography. ${ }^{60}$ With $580 \mathrm{~nm}$ height of grating bars, the first-order diffraction efficiency of TGs is $7 \%$ for $10 \mathrm{keV}$ hard $\mathrm{x}$-rays, which is sufficient for many applications. The testing results have highlighted the advantages of the combination of EBL, XRL, and UVL for fabricating $\mathrm{X}$-ray diffractive optical elements with a high lines density and a high aspect ratio.

\section{Conclusions}

Two combined lithographic methods have been developed for fabricating high-line-density x-ray TGs with a vertical cross section. The former one, combined by EBL and optical lithography, is able to individually fabricate $\mathrm{x}$-ray TGs with extremely high line density. The later one consisting of EBL, XRL, and UVL can manufacture $\mathrm{X}$-ray TGs in high throughput. To realize X-ray TGs with a line density of up to 6666 lines/mm, EBL was performed to directly pattern grating structures on polyimide membrane, which can effectively suppress the proximity effect caused by the forward and backscattered electrons. Thus, the cross section can be defined to be vertical, and the feature size of grating bars was down to $65 \mathrm{~nm}$. The relative diffraction efficiency in a photon energy range of 2.1 to $4.0 \mathrm{keV}$ was achieved as $8.89 \%$ to $9.41 \%$. Aiming at heavy demands of x-ray TGs, EBL, XRL, and UVL were combined to fabricate X-ray TGs with line density of up to 5000 limes $/ \mathrm{mm}$ in high throughput and in high fidelity. X-ray diffraction effect between mask and wafer was simulated by a 1-D FFT-BPM method. It demonstrated that the gap of mask/wafer must be kept within $3 \mu \mathrm{m}$; thus, one can obtain a high-aspect-ratio structures with feature size of down to $100 \mathrm{~nm}$ and height of up to $2 \mu \mathrm{m}$. Using the straightforward process, x-ray TGs with a line density of 5000 lines $/ \mathrm{mm}$ were fabricated in volume production. The characterization results have implied that the ratio of grating bar and space was controlled as 0.44 , definitely close to the optimal value of 0.5 . More importantly, both the former and the later combined methods are able to not only construct nanoscale and high-aspect ratio structures but also generate arbitrary patterns, providing more flexibility and design freedom for x-ray diffractive optical elements and promoting the development of x-ray scientific instruments.

\section{Acknowledgments}

We would like to extend our gratitude to Professor Gang Liu (USTC) and Futing Yi (IHEP, CAS) for their help on X-ray lithography, senior engineer Hongjun Zhou (USTC) and Professor Mingqi Cui (IHEP, CAS) for their help on testing, and Mr. Matteo Todeschini (Danchip, DTU) for his a ssistance in grammar. This work was supported by the National Key Research and Development Program of China (2017YFA0206002) and China Scholarship Council (201504910112).

\section{References}

1. V. Burwitz et al., "First high-resolution Chandra LETGS spectrum of the transient supersoft X-ray source RX J0513.9-6951," Adv. Space Res. 40, 1294-1298 (2007).

2. K. A. Flanagan et al., "Chandra high-resolution X-ray spectrum of supernova remnant 1E 0102.2-7219," Astrophys. J. 605, 230-246 (2004).

3. D. R. McMullin et al., "Extreme-ultraviolet efficiency measurements of freestanding transmission gratings," Appl. Opt. 43, 3797 (2004).

4. H. Wen et al., "Subnanoradian X-ray phase-contrast imaging using a far-field interferometer of nanometric phase gratings," Nat. Commun. 4, 2659 (2013).

5. F. Pfeiffer et al., "Phase retrieval and differential phase-contrast imaging with low-brilliance x-ray sources," Nat. Phys. 2, 258-261 (2006).

6. F. Pfeiffer et al., "Hard-x-ray dark-field imaging using a grating interferometer," Nat. Mater. 7, 134-137 (2008).

7. N. Morimoto et al., "X-ray phase contrast imaging by compact TalbotLau interferometer with a single transmission grating," Opt. Lett. 39, 4297-4300 (2014).

8. A. S. Moore et al., "A soft X-ray transmission grating imaging-spectrometer for the National Ignition Facility," Rev. Sci. Instrum. 83, $10 \mathrm{E} 132$ (2012).

9. R. J. Leeper et al., "Target diagnostic system for the national ignition facility (invited)," Rev. Sci. Instrum. 68, 868-879 (1997).

10. N. M. Ceglio, A. M. Hawryluk, and R. H. Price, "Imaging X-ray spectrometer for laser fusion applications," Appl. Opt. 21, 3953-3960 (1982).

11. D. Kumar et al., "Dual transmission grating based imaging radiometer for tokamak edge and divertor plasmasa," Rev. Sci. Instrum. 83, 10E511 (2012).

12. T. A. Savas et al., "Achromatic interferometric lithography for 100-nmperiod gratings and grids," J. Vac. Sci. Technol. B 13, 2732 (1995).,

13. T. M. Bloomstein et al., "22-nm immersion interference lithography," Opt. Express 14, 6434 (2006)

14. M. Ahn, R. K. Heilmann, and M. L. Schattenburg, "Fabrication of $200 \mathrm{~nm}$ period blazed transmission gratings on silicon-on-insulator wafers," J. Vac. Sci. Technol. B 26, 2179-2182 (2008).

15. R. Divan et al., "Metal-assisted etching of silicon molds for electroforming," J. Vac. Sci. Technol. B 31, 06FF03 (2013).

16. D. Noda, A. Tokuoka, and T. Hattori, "Fabrication of Si mold using ICP etching for x-ray diffraction grating," Key Eng. Mater. 523-524, 587 (2012).

17. J. Lenz, T. Wilhein, and S. Irsen, "Nanofabrication of diffractive elements for soft $\mathrm{x}$-ray and extreme ultraviolet applications using ion beam lithography," Appl. Phys. Lett. 95, 191118 (2009).

18. J. Zhang et al., "Diffraction properties for 1000 lines/mm free-standing quantum-dot-array diffraction grating fabricated by focused ion beam," Chin. Phys. Lett. 31, 124204 (2014).

19. A. Holmberg, M. Lindblom, and H. M. Hertz, "Controlled electroplating for high-aspect-ratio zone plate fabrication," J. Vac. Sci. Technol. B 24, 2592 (2006).

20. S. Gorelick et al., "Direct e-beam writing of high aspect ratio nanostructures in PMMA: a tool for diffractive x-ray optics fabrication," Microelectron. Eng. 87, 1052-1056 (2010).

21. E. Chubarova et al., "Platinum zone plates for hard x-ray applications," Microelectron. Eng. 88, 3123 (2011). 
22. T. Greibe et al., "Quality control of JEOL JBX-9500FSZ e-beam lithography system in a multi-user laboratory," Microelectron. Eng. 155, 25-28 (2016).

23. J. Vila-Comamala et al., "Dense high aspect ratio hydrogen silsesquioxane nanostructures by $100 \mathrm{keV}$ electron beam lithography," Nanotechnology 21, 285305 (2010).

24. H. Duan et al., "Metrology for electron-beam lithography and resist contrast at the sub-10 nm scale," J. Vac. Sci. Technol. B 28, C6H11 (2010).

25. J. K. W. Yang et al., "Understanding of hydrogen silsesquioxane electron resist for sub-5-nm-half-pitch lithography," J. Vac. Sci. Technol. B 27, 2622 (2009).

26. S. Gorelick et al., "High aspect ratio nanostructuring by high energy electrons and electroplating," Microelectron. Eng. 88, 2259-2262 (2011).

27. S. J. Goh et al., "Fabrication and characterization of freestanding, high-line-density transmission gratings for the vacuum UV to soft X-ray range," Opt. Express 23, 4421-4434 (2015).

28. S. Gorelick et al., "High-efficiency gold Fresnel zone plates for multikeV x-rays," AIP Conf. Proc. 1365, 88 (2011).

29. C. R. Canizares et al., "The chandra high energy transmission grating: design, fabrication, ground calibration and five years in flight," $\mathrm{Publ}$. Astron. Soc. Pac. 117, 1144-1171 (2005)

30. B. L. Henke, E. M. Gullikson, and J. C. Davis, "X-ray interactionsphotoabsorption, scattering, transmission and reflection at $\mathrm{E}=50-$ $30000 \mathrm{eV}, Z=1-92$," At. Data Nucl. Data Tables 54, 181-342 (1993).

31. G. K. Skinner, "Diffractive X-ray telescopes," X-Ray Opt. Instrum. 2010, 743485 (2010).

32. T. Weitkamp et al., "X-ray wavefront analysis and optics characterization with a grating interferometer," Appl. Phys. Lett. 86, 054101 (2005).

33. N. M. Ceglio et al., "Time-resolved X-ray transmission grating spectrometer for studying laser-produced plasmas," Appl. Opt. 22, 318 (1983).

34. H. W. Schnopper et al., "Diffraction grating transmission efficiencies for XUV and soft x rays," Appl. Opt. 16, 1088 (1977).

35. T. H. Markert et al., "Modeling the diffraction efficiencies of the AXAF high energy transmission gratings," Proc. SPIE 2518, 427 (1995).

36. M. C. Hettrick et al., "Profiled bar transmission gratings: soft-x-ray calibration of new Kirchoff solutions," Appl. Opt. 43, 3772 (2004).

37. A. M. Hawryluk et al., "Gold transmission gratings with submicrometer periods and thicknesses $\gtrsim 0.5 \mu \mathrm{m}, " J$. Vac. Sci. Technol. B 19(4), 897900 (1981).

38. M. L. Schattenburg et al., "Fabrication of high energy x-ray transmission gratings for AXAF," Proc. SPIE 2280, 181 (1994).

39. H. Brauninger et al., "Fabrication of transmission gratings for use in cosmic x-ray and XUV astronomy," Appl. Opt. 18(20), 3502-3505 (1979).

40. T. A. Savas et al., "Large-area achromatic interferometric lithography for $100 \mathrm{~nm}$ period gratings and grids," J. Vac. Sci. Technol. 14(6), 4167 (1996).

41. M. L. Schattenburg, E. H. Anderson, and H. I. Smith, "X-ray/VUV transmission gratings for astrophysical and laboratory applications," Phys. Scr. 41, 13-20 (1990).

42. A. Bruccoleri et al., "Fabrication of nanoscale, high throughput, high aspect ratio freestanding gratings," J. Vac. Sci. Technol. B 30, 06FF03 (2012).
43. L. Wang et al., "High-resolution nanopatterning by achromatic spatial frequency multiplication with electroplated grating structures," J. Vac. Sci. Technol. B 30, 031603 (2012).

44. B. Paivanranta et al., "Sub-10 nm patterning using EUV interference lithography," Nanotechnology 22, 375302 (2011).

45. L. Wang, H. H. Solak, and Y. Ekinci, "Fabrication of high-resolution large-area patterns using EUV interference lithography in a scan-exposure mode," Nanotechnology 23, 305303 (2012).

46. H. H. Solaka et al., "Sub-50 nm period patterns with EUV interference lithography," Microelectron. Eng. 67-68, 56 (2003).

47. D. Fan et al., "Patterning of nanodot-arrays using EUV achromatic Talbot lithography at the Swiss Light Source and Shanghai Synchrotron Radiation Facility," Microelectron. Eng. 155, 55-60 (2016).

48. W. Wu et al., "Sub-10 nm nanoimprint lithography by wafer bowing," Nano Lett. 8(11), 3865-3869 (2008).

49. W. Yashiro et al., "A metallic glass grating for x-ray grating interferometers fabricated by imprinting," Appl. Phys. Exp. 7, 032501 (2014).

50. C. Chang and A. Sakdinawat, "Ultra-high aspect ratio high-resolution nanofabrication for hard x-ray diffractive optics," Nat. Commun. 5, 4243 (2014).

51. Y. Liu et al., "Quasi suppression of higher order diffractions with inclined rectangular apertures gratings," Sci. Rep. 5, 16502 (2015).

52. J. Liu et al., "Simulation and experimental study of aspect ratio limitation in Fresnel zone plates for hard-X-ray optics," Appl. Opt. 54, 9630 (2015).

53. C. Xie et al., "Fabrication of x-ray diffractive optical elements for laser fusion applications," Opt. Eng. 52, 033402 (2013).

54. D. Wang et al., "Microzone plates with high-aspect ratio fabricated by e-beam and x-ray lithography," J. Microlith., Microfab., Microsyst. 5(1), 013002 (2006).

55. D. Noda et al., "Fabrication of x-ray gratings using x-ray lithography technique for x-ray talbot interferometer," J. Electrochem. Soc. 156(5), H299 (2009).

56. D. Noda et al., "Fabrication of large area diffraction grating using LIGA process," Microsyst. Technol. 14, 1311-1315 (2008).

57. E. Toyota and M. Washio, "Extendibility of proximity x-ray lithography to $25 \mathrm{~nm}$ and below," J. Vac. Sci. Technol. B 20, 2979 (2002).

58. S. B. Bollepalli, M. Khan, and F. Cerrina, "Image formation in extreme ultraviolet lithography and numerical aperture effects," J. Vac. Sci. Technol. 17, 2992 (1999).

59. J. Xiao et al., "Modeling image formation: application to mask optimization," J. Vac. Sci. Technol. B 12, 4038 (1994).

60. P. Desaute et al., "Characterization of a high resolution transmission grating," Opt. Commun. 173, 37-43 (2000).

Xiaoli Zhu is an associate professor in the Institute of Microelectronics of Chinese Academy of Sciences. He received his BS degree in applied physics from Lanzhou University in 1999, his MS degree in optics from Beijing University of Technology in 2005, and his PhD in microelectronics from the Institute of Microelectronics of Chinese Academy of Sciences in 2008. His current research interests include nanoscale lithography, micro- and nanofabrication, X-Ray and EUV diffractive optical elements, and their applications.

Biographies for the other authors are not available. 